\title{
MicroRNA-375 inhibits lung surfactant secretion by altering cytoskeleton reorganization
}

Honghao Zhang, Amarjit Mishra, Narendranath Reddy Chintagari, Deming Gou, and Lin Liu Lundberg-Kienlen Lung Biology and Toxicology Laboratory, Department of Physiological Sciences, Oklahoma State University, Stillwater, Oklahoma, 74078

\begin{abstract}
Lung surfactant is secreted via exocytosis of lamellar bodies from alveolar epithelial type II cells. Whether microRNAs regulate lung surfactant secretion is unknown. MicroRNA-375 (miR-375) has been shown to be involved in insulin secretion. In this paper, we report that the overexpression of miR-375 inhibited lung surfactant secretion. However, miR-125a, miR-30a, miR-1, miR-382 and miR-101 did not influence lung surfactant secretion. miR-375 had no effects on surfactant synthesis or the formation of lamellar bodies. However, miR-375 did abolish the lung surfactant secretagogue-induced disassembly and reassembly of cytoskeleton. Our results suggest that miR-375 regulates surfactant secretion via the reorganization of cytoskeleton.
\end{abstract}

\section{Keywords}

miR-375; lung surfactant secretion; cytoskeleton; type II cells

\section{Introduction}

\begin{abstract}
Alveoli are the basic units for gas exchange. Alveolar epithelial type II cells secrete lung surfactant, a surface active material to reduce surface tension at the air-liquid interface in the alveoli. Lung surfactant is synthesized in the endoplasmic reticulum of the alveolar type II cells before they are packed, transported and finally stored in lamellar bodies. Lamellar bodies eventually fuse with the plasma membrane to release surfactant once induction signals are received [1]. Efforts in our laboratory have been carried out continuously to uncover the mechanisms of surfactant secretion by identifying the key components involved in the fusion of lamellar bodies with the plasma membrane. The t-SNARE proteins, SNAP-23 and Syntaxin 2, and the SNARE associated proteins, NSF and $\alpha$-SNAP are present in type II cells and are required for lung surfactant secretion [2;3]. By collaborating with arachidonic acid, annexin $\mathrm{A} 2\left(\mathrm{a} \mathrm{Ca}^{2+}\right.$ sensor) is responsible for driving the fusion between lamellar bodies and the plasma membrane [4]. Annexin A2 also interacts with SNAP-23 [5]. In addition, the lipid raft on the type II cell membrane has been demonstrated to be the microdomains where the lamellar bodies dock, the fusion pore forms, and the membrane fusion takes place [6].
\end{abstract}

MicroRNAs (miRNAs) are small non-coding RNAs with regulatory functions of gene expression. In mammals, miRNA is transcribed by RNA polymerase II to pri-miRNA and processed by drosha to pre-miRNA by trimming off its flanking sequences. Pre-miRNA is transported into cytoplasm by exportin5 and cleaved by Dicer in cytoplasm to 20 bp RNA

Address correspondence to: Lin Liu, Department of Physiological Sciences, Oklahoma State University, 264 McElroy Hall, Stillwater, OK 74078, Tel. 405 744-4526, Fax. 405 744-8263, lin.liu@ okstate.edu. 
duplexes [7;8]. In most cases, one strand is completely degraded and the other strand binds with several other proteins to form the complex. Based on complementary base pairing, the complex selectively binds to the 3'-UTR region of mRNA to initiate translational repression or mRNA cleavage.

More than 700 miRNAs are documented in the human genome. It is predicted that miRNAs are involved in the regulation of almost every cellular and physiological process. The specific biological and physiological function of each miRNA is gradually understood. However, it is still far away from complete. In this study, through our effort in searching for miRNAs involved in the regulation of surfactant secretion in alveolar type II cells, we identified miR-375 as a negative regulator of this process. Furthermore, we found that miR-375 regulates surfactant secretion by inhibiting cytoskeleton reorganization.

\section{Materials and Methods}

\section{Reagents}

Fetal bovine serum (FBS), trypsin-EDTA, Dulbecco's modified Eagle's medium (DMEM), Opti-MEM, nonessential amino acids (NEAA) and Lipofactamine 2000 were purchased from Invitrogen Life Technologies (Carlsbad, CA). Enhanced chemilluminescence (ECL) reagent was from Amersham Pharmacia Biotech (Arlington Heights, IL). Oligos were synthesized by Sigma-genosys at 0.025 scales. 18S rRNA primers were from Ambion (Austin, TX). Restriction enzymes were from NewEngland Biolab, unless mentioned otherwise. Ligase for cloning was from Invitrogen. Enzymes for polymerase chain reaction (PCR) were purchased from Genscript. Monoclonal anti-ABCA3 (LB-180) antibodies were from Covance Research products (Richmond, CA). Polyclonal goat anti-SP-C antibodies were from Santa Cruz Biotechnology (Santa Cruz, CA). Reagents and primers for miRNA real-time PCR was obtained from Applied Biosystem. HEK 293A cells (Invitrogen) were cultured in D-MEM with $10 \%(\mathrm{v} / \mathrm{v})$ FBS, $1 \%$ (v/v) NEAA.

\section{Construction of adenoviral miRNA overexpression vectors}

Mature miRNAs with flanking sequences ( $200 \mathrm{bp}$ at each end) were amplified using primers listed in Table I from human or rat genomic DNAs, followed by digestion with Sal I and Eco RI. The digested PCR product was cloned into a modified pENTR/CMV-EGFP plasmid between EGFP and VS40 poly A terminal sequence. The empty vector without a miRNA insert was used as a vector control. The insert in the pENTR vector was switched into an adenoviral vector by the Gateway technique (Invitrogen, Carlsbad, CA), which was linearized by $\mathrm{PacI}$. Adenovirus was produced by transfecting the adenoviral vector into HEK 293A cells and amplified by transducing HEK 293A cells. Virus titer was assayed by making dilutions of viral stock, transducing HEK 293A cells and counting virus-infected cells.

\section{miRNA real-time PCR}

Total RNAs were isolated by using the mirVana miRNA isolation kit (Ambion). $100 \mathrm{ng}$ of total RNAs were used in reverse transcription for miR-375 or 18s RNA. MiR-375 was measured by real-time PCR using the TaqMan microRNA assay kit (Applied Biosystem).

\section{Alveolar type II cells isolation and culture}

Alveolar type II cells were isolated from Sprague-Dawley male rats (180-200 g) as previously described [9]. Rats were anesthetized intraperitoneally with a mixture of ketamine and xylazine. Lungs were perfused, lavaged and digested with elastase. Lung tissues were chopped and filtered. The cells were subjected for IgG panning to remove 
macrophages. The unattached cells were pelleted and resuspended. Cell viability was $>97 \%$ as determined by the trypan-blue exclusion assay. The purity was $>90 \%$.

\section{Surfactant secretion assay}

Alveolar type II cells $\left(1 \times 10^{6}\right)$ were seeded on $35 \mathrm{~mm}$ dishes and labeled with $0.6 \mu \mathrm{Ci}$ of $\left[{ }^{3} \mathrm{H}\right]$ choline. If needed, adenoviruses were added at the time when cells were plated. After overnight culture, the unattached cells were removed. The attached cells were washed with warm MEM and incubated in fresh media for $30 \mathrm{~min}$. Then, cells were stimulated by $0.1 \mu \mathrm{M}$ phorbol 12-myristate 13-acetate, $100 \mu \mathrm{M}$ ATP, and $20 \mu \mathrm{M}$ terbutaline for $2 \mathrm{~h}$. Surfactant secretion assay was done as previously described [9].

\section{Immunofluorescence}

Freshly isolated alveolar type II cells were plated on cover slips. Adenoviruses were added at the time of plating. After a 18-h incubation, cells were fixed with $4 \%$ paraformaldehyde for $20 \mathrm{~min}$ at room temperature. Then, cells were permeabilized with $1 \%$ Triton X-100 and blocked by $10 \%$ FBS in $50 \mathrm{mM}$ PBS. Cells were incubated with anti-ABCA3 (1:500) or anti-SP-C $(1: 100)$ antibodies at $37^{\circ} \mathrm{C}$ for $2 \mathrm{~h}$, followed by washing in PBS for 3 times for 5 min each. Cells were incubated with Alexa Fluor 568 anti-mouse or anti-rabbit IgG for $2 \mathrm{~h}$ at $37^{\circ} \mathrm{C}$. Finally, the slides were washed, mounted and examined under a fluorescence microscopy.

\section{Results}

To investigate the roles of miRNA in surfactant secretion, we constructed adenoviral vectors for the overexpression of miRNAs. This vector encodes enhanced green fluorescence protein (eGFP) between miRNA sequence and CMV promoter. Hence, transfection efficiency can be monitored by examination of expressed eGFP. Mature miRNAs and flanking sequences were amplified from human or rat genome. An adenovirus expressing miR-375 was used to transduce primary alveolar type II cells. At $18 \mathrm{hrs}$ post transfection, almost every cell had positive expression of eGFP (Fig. 1A), indicating that the transduction of the adenovirus into alveolar type II cells reached nearly $100 \%$. To examine whether miR-375 is overexpressed in type II cells, total RNAs were isolated and mature miR-375 level was measured by real-time PCR. As shown in Fig. 1B, compared to virus control, adenovirus vector for overexpression of miR-375 gave a significant higher expression level of miR-375. An adenoviral vector expressing rat mature miR-375 and flanking sequences had a comparable miR-375 expression as the human miR-375 vector. The human miRNA expression vectors were used in all of the subsequent experiments.

Our system to deliver and overexpress miRNAs in primary type II cells worked as expected. We then used this system to screen miRNAs potentially involved in the regulation of surfactant secretion in alveolar type II cells. Our previous work has demonstrated that the expression of miR-375, miR-125a, miR-30a, miR-1, miR-382, and miR-101 are detected in the lung [10]. We overexpressed each of these miRNAs in alveolar type II cells by using the adenoviral vectors. As shown in Fig. 2, the overexpression of miR-375 led to a significant decrease in surfactant secretion in comparison with virus control. All of the other miRNAs had no effects.

To explore the possible mechanism of how miR-375 exerts its effect, we first determined if miR-375 was involved in surfactant synthesis. For this purpose, immunocytochemistry was performed to examine surfactant protein C (SP-C). The overexpression of miR-375 resulted in no change in the expression level of SP-C in alveolar type II cells (Fig. 3). Similarly, we also determined whether miR-375 impairs the formation of lamellar bodies, secretory 
granules in type II cells. The overexpression of miR-375 in alveolar type II cells had no effect on the number of lamellar bodies as determined by staining lamellar bodies with antiABCA3 (a lamellar body limiting membrane protein) antibodies (Fig. 3). The virus control or miR-101 did not affect SP-C nor ABCA3 expression.

Our previous work has demonstrated that upon stimulation of alveolar type II cells, cortical cytoskeleton undergoes disassembly and reassembly to allow a lamellar body to access and fuse with the plasma membrane [11]. We wondered if miR-375 would affect F-actin disassembly and reassembly. For this purpose, type II cells were stimulated with terbutaline and fixed at different time points. Alexa Fluor-568 Phalloidin was used to label F-actin. As shown in Fig. 4, in blank control cells and virus control-treated cells, upon stimulation, the disassembly of F-actin occurred at 3 min and the reassembly of F-actin was observed at 5$10 \mathrm{~min}$. However, in the miR-375-overexpressed cells, no reassembly of F-actin was observed within $10 \mathrm{~min}$. This effect is specific since miR-101 had no effects on the reassembly of F-actin. This result clearly demonstrates that the overexpression of miR-375 inhibits the reassembly of F-actin.

\section{Discussion}

miRNAs are important regulatory molecules. Bioinformatics predicts that $\sim 30 \%$ of mammalian protein-coding genes are potentially under the regulation of miRNAs. Accordingly, miRNAs are suggested to be involved in regulating almost every cellular and physiological process [7;12]. Changes in their expression are associated with almost every type of human pathology [12]. In searching for miRNAs that potentially regulate lung surfactant secretion, we identified miR-375 as a negative regulator of this process. Furthermore, we demonstrated that the overexpression of miR-375 interfered with cortical cytoskeleton reassembly upon the stimulation of type II cells with lung surfactant secretagogues.

In pancreatic $\beta$-cells, the overexpression of miR-375 leads to improper docking of secretory granules with the plasma membrane. However, neither granules density, nor actin filament networks are affected [13]. In our system, when miR-375 was overexpressed in type II cells, we did not find changes in surfactant synthesis or in the number of lamellar bodies. Previous work in our laboratory demonstrates that cortical actin cytoskeleton acts as a barrier to prevent the fusion of secretion vesicles with the plasma membrane [11]. Upon the stimulation of type II cells, the rearrangement of cytoskeleton initiates from a transient disassembly of F-actin, followed by reassembly of the cortical cytoskeleton so that the translocation of secretory vesicles occurs. When miR-375 was overexpressed in alveolar type II cells, the reassembly of F-actin was not observed within 10 minutes. Failure to form proper actin network for motor-mediated translocation of secretory vesicles may ultimately hamper proper docking and fusion of secretory vesicles with the plasma membranes.

The mechanisms through which miR-375 regulates reorganization of cytoskeleton remain to be determined. However, the expression level of myotrophin, one of the direct targets of miR-375, significantly decreases in the miR-375-overexpresseed pancreatic $\beta$-cells [13]. Myotropin, as reported, is capable of interaction with the actin binding protein, Cap Z, which caps the barbed end of actin filaments and mediates nucleation of actin polymerization [14-17]. Through the interaction with CapZ, myotropin may regulate actin polymerization although the detailed mechanism is not known yet. When miR-375 is overexpressed, myotropin is reduced. As such, there is no enough cytosolic myotropin available to interact with CapZ. Thus, it can not initiate actin polymerization following Factin disassembly. As a result, no enough actin network becomes available for the 
translocation of secretory vesicles. In summary, we present evidence that miR-375 is a negative regulator of surfactant secretion by inhibiting reassembly of cortical cytoskeleton.

\section{Acknowledgments}

This work was supported by NIH R01 HL-083188, R01 HL-052146, and R01 HL-071628 (To LL).

\section{References}

1. Andreeva AV, Kutuzov MA, Voyno-Yasenetskaya TA. Regulation of Surfactant Secretion in Alveolar Type II cells. Am.J Physiol Lung Cell Mol.Physiol. 2007; 293:L259-L271. [PubMed: 17496061]

2. Abonyo BO, Gou D, Wang P, Narasaraju T, Wang Z, Liu L. Syntaxin 2 and SNAP-23 are required for regulated surfactant secretion. Biochemistry. 2004; 43:3499-3506. [PubMed: 15035620]

3. Abonyo BO, Wang P, Narasaraju TA, Rowan WH III, McMillan DH, Zimmerman UJ, Liu L. Characterization of alpha-Soluble N-Ethylmaleimide-Sensitive Fusion Attachment Protein in Alveolar Type II Cells: Implications in Lung Surfactant Secretion. Am.J.Respir.Cell Mol.Biol. 2003; 29:273-282. [PubMed: 12663329]

4. Liu L, Fisher AB, Zimmerman UJP. Lung annexin II promotes fusion of isolated lamellar bodies with liposomes. Biochim.Biophys.Acta. 1995; 1259:166-172. [PubMed: 7488637]

5. Wang P, Chintagari NR, Gou D, Su L, Liu L. Physical and Functional Interactions of SNAP-23 with Annexin A2. Am J Respir Cell Mol Biol. 2007; 37:467-476. [PubMed: 17575076]

6. Chintagari NR, Jin N, Wang P, Narasaraju TA, Chen J, Liu L. Effect of cholesterol depletion on exocytosis of alveolar type II cells. Am J Respir.Cell Mol Biol. 2006; 34:677-687. [PubMed: 16439800]

7. Bushati N, Cohen SM. microRNA Functions. Annu.Rev.Cell Dev.Biol. 2007; 23:175-205. [PubMed: 17506695]

8. Rana TM. Illuminating the silence: understanding the structure and function of small RNAs. Nat.Rev.Mol.Cell Biol. 2007; 8:23-36. [PubMed: 17183358]

9. Liu L, Wang M, Fisher AB, Zimmerman UJP. Involvement of annexin II in exocytosis of lamellar bodies from alveolar epithelial type II cells. Am.J.Physiol. 1996; 270:L668-L676. [PubMed: 8928828]

10. Wang Y, Weng T, Gou D, Chen Z, Chintagari NR, Liu L. Identification of rat lung-specific microRNAs by microRNA microarray: valuable discoveries for the facilitation of lung research. BMC.Genomics. 2007; 8:29. [PubMed: 17250765]

11. Singh TK, Abonyo B, Narasaraju TA, Liu L. Reorganization of cytoskeleton during surfactant secretion in lung type II cells: a role of annexin II. Cell Signal. 2004; 16:63-70. [PubMed: 14607276]

12. Esquela-Kerscher A, Slack FJ. Oncomirs - microRNAs with a role in cancer. Nat.Rev.Cancer. 2006; 6:259-269. [PubMed: 16557279]

13. Poy MN, Eliasson L, Krutzfeldt J, Kuwajima S, Ma X, Macdonald PE, Pfeffer S, Tuschl T, Rajewsky N, Rorsman P, Stoffel M. A pancreatic islet-specific microRNA regulates insulin secretion. Nature. 2004; 432:226-230. [PubMed: 15538371]

14. Taoka M, Ichimura T, Wakamiya-Tsuruta A, Kubota Y, Araki T, Obinata T, Isobe T. V-1, a protein expressed transiently during murine cerebellar development, regulates actin polymerization via interaction with capping protein. J Biol.Chem. 2003; 278:5864-5870. [PubMed: 12488317]

15. Caldwell JE, Heiss SG, Mermall V, Cooper JA. Effects of CapZ, an actin capping protein of muscle, on the polymerization of actin. Biochemistry. 1989; 28:8506-8514. [PubMed: 2557904]

16. Maruyama K, Kurokawa H, Oosawa M, Shimaoka S, Yamamoto H, Ito M, Maruyama K. Betaactinin is equivalent to Cap Z protein. J Biol.Chem. 1990; 265:8712-8715. [PubMed: 2341404]

17. Casella JF, Maack DJ, Lin S. Purification and initial characterization of a protein from skeletal muscle that caps the barbed ends of actin filaments. J Biol.Chem. 1986; 261:10915-10921. [PubMed: 3733738] 
Bright field

A

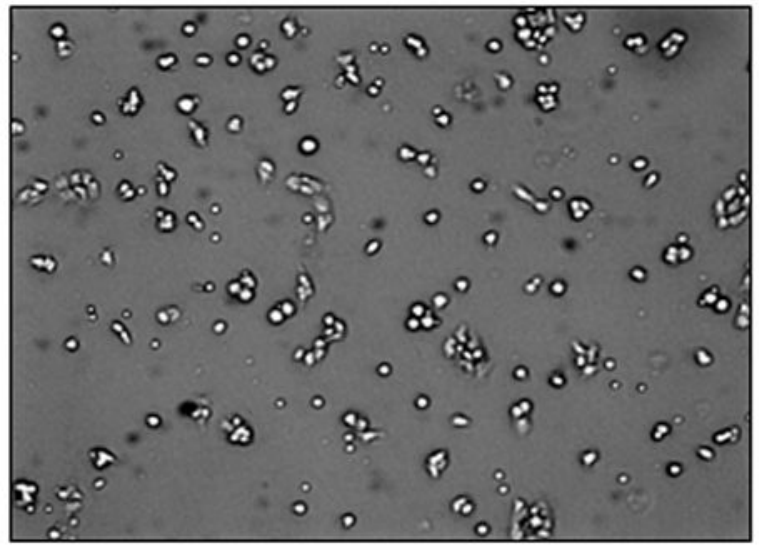

GFP

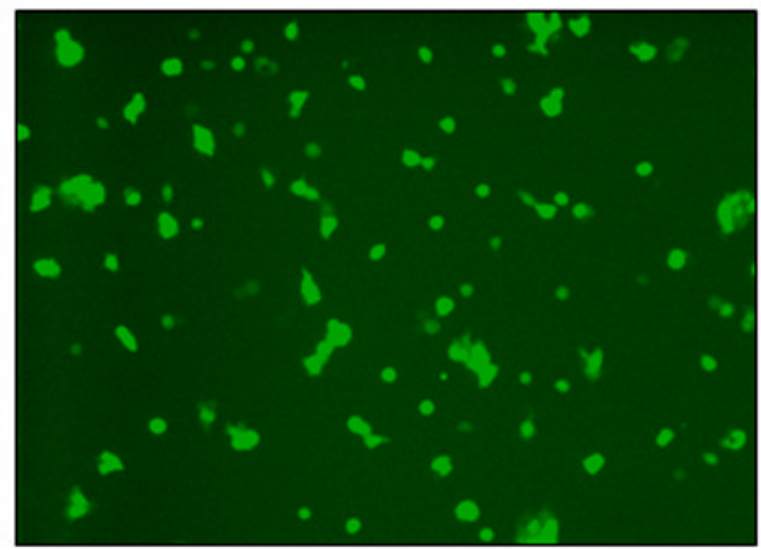

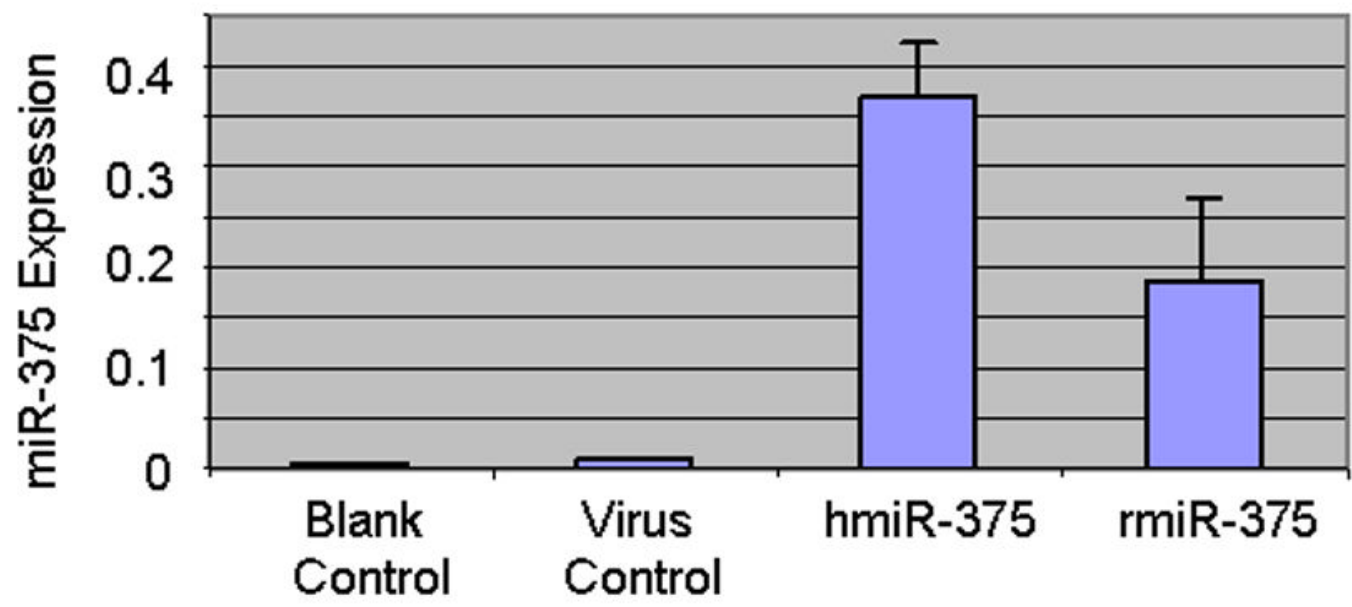

Fig. 1. Overexpression of miR-375 in alveolar type II cells

Type II cells were transduced with none (blank control) or adenoviruses carrying an empty vector (virus control), a human miR-375 (hmiR-375) or a rat miR-375 (rmiR-375), at a MOI of 75. At 24 hours post-transduction, GFP fluorescence was observed (A, hmiR-375) and miR-375 levels were measured (B). The miR-375 expression levels were normalized to $18 \mathrm{~S}$

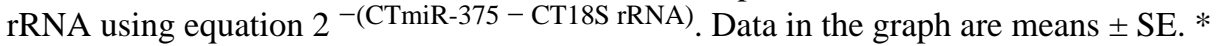
$\mathrm{P}<0.05$ v.s. virus control, $\mathrm{n}=3$. 


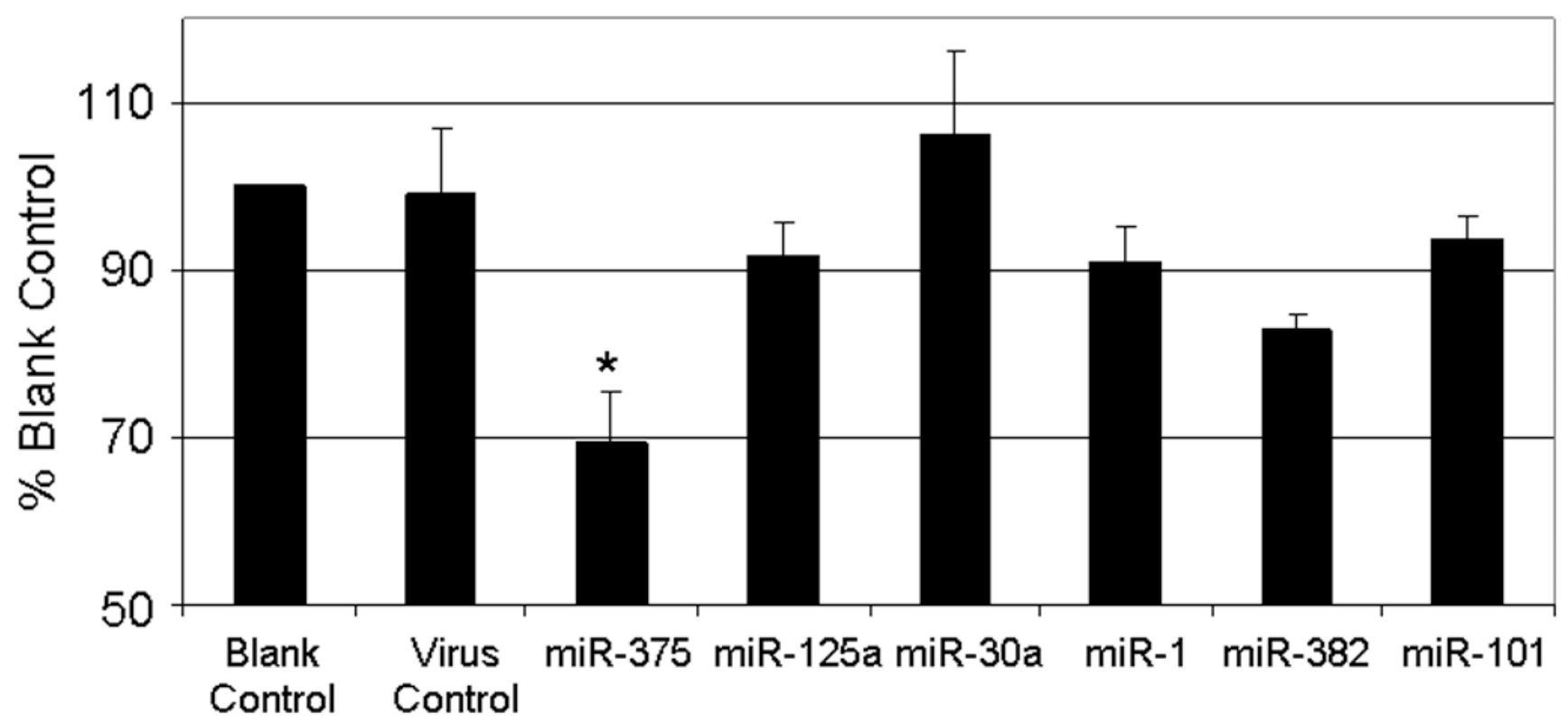

Fig. 2. Effects of overexpression of miR-375 on surfactant secretion Adenoviruses carrying human miRNAs were added to type II cells at a MOI of 75. After a 24-hr culture, the cells were stimulated and surfactant secretion was assayed. Data shown are percentages of blank control. Error bars stand for standard error. $* \mathrm{P}<0.05$ v.s. control. $\mathrm{n}=6$ for $\mathrm{miR}-375 ; \mathrm{n}=3$ for the rest. 


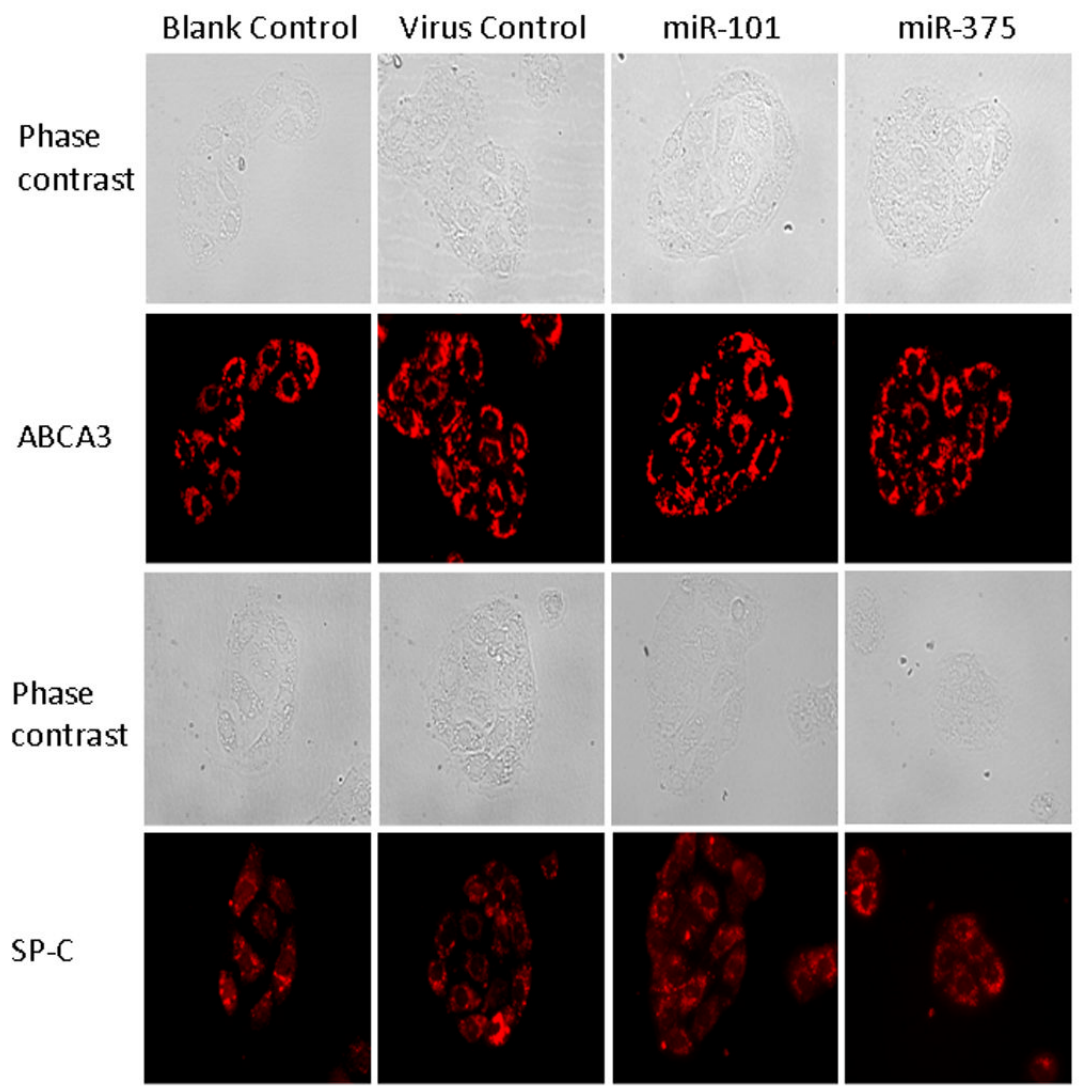

Fig. 3. Effect of miR-375 on SP-C and ABCA3 expression

Alveolar type II cells were incubated overnight without (blank control) or with adenoviruses carrying an empty vector (virus control), human miR-375 or human miR-101 at a MOI of 75. Cells were immunostained with either anti-SP-C or anti-ABCA3 antibodies. 


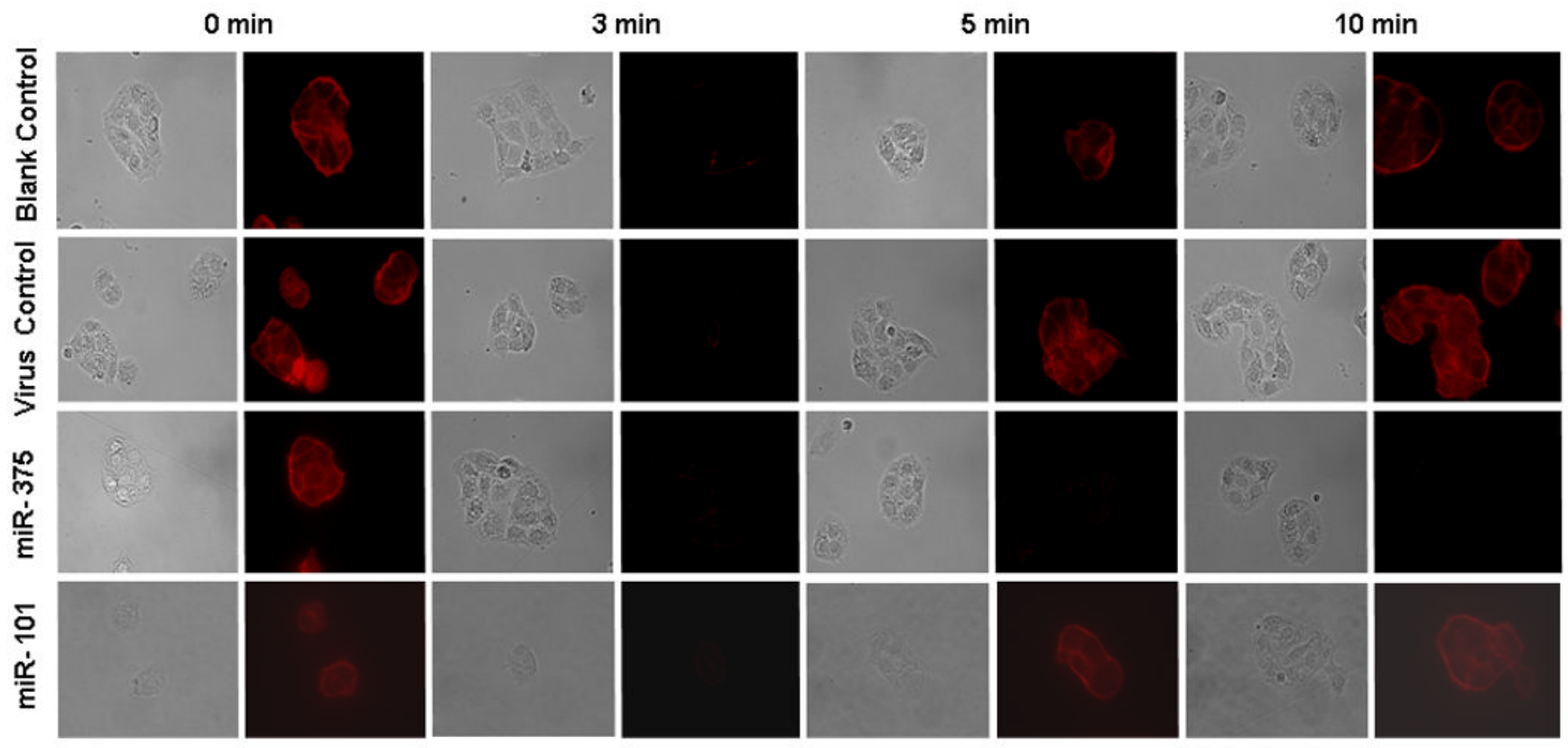

Fig. 4. Effect of miR-375 on cytoskeleton reorganization

Alveolar type II cells were incubated overnight without adenovirus (blank control) or with adenoviruses carrying an empty vector (virus control), human miR-375 or human miR-101 at a MOI of 75. Then, cells were stimulated with $10 \mu \mathrm{M}$ terbutaline for $0 \mathrm{~min}, 3 \mathrm{~min}, 5 \mathrm{~min}$, and $10 \mathrm{~min}$. At the end of incubation, cells were fixed and stained with $50 \mathrm{nM}$ Alexa Fluor-568 Phalloidin. Phase contrast and fluorescence images were taken. 
Table 1

Primers for miRNA expression vectors

\begin{tabular}{|l|l|l|}
\hline & Foward Primer & Reverse Primer \\
\hline hsa-miR-375 & cacctcgagGCACAGCCTCTCCCACCCGTA & gagaattcCGTGTCAGCCGCAGATGCGT \\
\hline rno-miR-375 & cacctcgaGCAGAAACTCCGTGGCGGGTGACCT & gagaattcTGGAGCCTGCTCAGCCCGCATTACC \\
\hline hsa-miR-125a & cacctcgagCCCGATATCTCTCTGTGTCTCTATTTC & gagaattcGGTCAGGTTTCAGTTGGTGGTC \\
\hline hsa-miR-30a & cacctcgAGGCTTCAGTACTTTACAGAATCG & gagaattcGGAAATATTGCCCTACTACGC \\
\hline hsa-miR-1 & cacctcgaGCTTAGGAATGTCTATGTCCACC & gagaaattcGCCAAAGGTCATCTGTTCATG \\
\hline hsa-miR-382 & cacctcgagCTGCCTCACTAATGCTCGGTG & gagaattCAGCTAGGTCAGCGCATTGTC \\
\hline hsa-miR-101 & cacctcgagTTTCTTCTGCCTCCTCACGTC & gagaattcGGCTGCACCAACAACTACCC \\
\hline
\end{tabular}

